\title{
Mise au point d'un modèle de simulation de la rouille brune du blé
}

\author{
E Benizri 1, F Projetti 2 \\ 1 Université Paul Sabatier, laboratoire de cryptogamie,118, route de Narbonne, 31062 Toulouse Cedex; \\ 2 Service régional de la protection des végétaux; BP 19, rue St-Jean-Prolongée, 31131 Balma, France
}

(Reçu le 9 avril 1991; accepté le 4 octobre 1991)

\begin{abstract}
Résumé - Cette étude présente un modèle de simulation de la rouille brune du blé, dont l'agent pathogène, Puccinia recondita f sp tritici, est le principal parasite des parties aériennes du blé dans le Sud-Ouest de la France. Le système mis en forme est un modèle déterministe, qui simule l'ensemble du cycle de l'agent pathogène. II utilise des données climatiques trihoraires (température et humectation) pour simuler les différentes phases de l'évolution de la maladie et intègre certains paramètres d'ordre phénologique et agronomique. Le modèle a été validé sur plusieurs années. La comparaison des courbes d'évolution des pustules infectieuses réelles et simulées montre globalement que ce système permet aujourd'hui de simuler correctement l'évolution de l'agent pathogène. Ainsi, de par sa conception et son organisation, il pourra être utilisé en tant qu'indicateur de risques et contribuer à l'amélioration des avertissements agricoles, mais aussi en tant que modèle d'aide à la décision à la parcelle dans le cadre notamment du raisonnement des interventions fongicides et de l'interprétation des résultats d'expérimentations des produits phytosanitaires.
\end{abstract}

rouille brune / Puccinia recondita / modélisation / blé

Summary - A model of simulation of wheat leaf rust. Puccinia recondita $f s p$ tritici is the most important disease of wheat in the south-west of France. The authors propose a model of simulation which is an indicator model of risk. It makes it possible to simulate the evolution of the wheat leaf rust (figs 1, 2). It uses climatic data to simulate various phases of the evolution of the disease; temperature and free moisture (fig 3) seem to be the main limiting factors in the infectious process for our regions. It also quantifies the stages of development of the fungus (figs 4, 5). This method has been tested for several years. A good correlation between the simulations of disease by the model and the field observations has been shown (fig 6). It can therefore be useful for fungicide trials as well as for diffusion of agricultural forecasts.

leafs rust / Puccinia recondita / modeling / simulation / wheat

\section{INTRODUCTION}

Parmi les maladies qui affectent le blé dans le Sud-Ouest de la France, la rouille brune, dont l'agent pathogène est Puccinia recondita Rob ex Desm f sp tritici Eriks et Henn, est préoccupante à 2 titres :

- elle trouve dans cette région au climat tempéré des conditions de maintien dans la sole céréalière qui en font une affection endémique et une menace économique quasiment permanente;

- elle peut, selon le climat printanier, s'exprimer certaines années avec violence et soudaineté de façon explosive et mettre en défaut les programmes de traitements basés sur le stade phénologique du blé.
Une prévision de la rouille brune s'impose si l'on veut rendre fiable la lutte fongicide dans la zone de prédilection de cette maladie.

La modélisation de la rouille brune a été travaillée par de nombreux auteurs. Dès 1946, Chester propose de considérer le «mois critique" (mars pour l'Oklahoma), c'est-à-dire celui où la température moyenne atteint $12^{\circ} \mathrm{C}$. L'utilisation de cette méthode permet de prévoir les années de fortes ou de faibles épidémies de rouille sur un plan qualitatif.

De nombreux modèles concernant la rouille brune, incluant des efforts de quantification, ont été ensuite mis en forme et le sont encore aujourd'hui. Certains d'entre eux sont établis par régression linéaire (Burleigh et al, 1972; Darles, 1980; Caron, 1988), d'autres, en se basant sur 
la loi des facteurs limitants (Waggoner et al, 1980; Poilane, 1982). Ces modèles sont dits descriptifs ou empiriques.

Une autre approche de la modélisation consiste à subdiviser l'épidémie en une succession de cycles unitaires d'infection qui sont euxmêmes décomposés en une série d'événements. De tels modèles sont dits déterministes, (Knudsen et al, 1987).

C'est avec cette démarche analytique que nous avons mis en forme un nouvel outil, dénommé EPURE (Étude du Puccinia recondita), (Benizri, 1989), en prenant pour base de travail le modèle SIMUL travaillé par Bova (1983). Les travaux de Bova reposaient sur le simulateur de Rijsdijk. Ce modèle utilisait pour ses calculs les données épidémiologiques de base de la rouille brune ainsi que des paramètres climatiques simples tels que la température moyenne journalière et la pluviométrie journalière. Ce modèle n'était pas totalement satisfaisant. En effet, les paramètres climatiques tels que la pluviométrie sont trop rustiques et donc pas assez précis pour intervenir dans les calculs du modèle, le système ne pouvait pas fonctionner sur une campagne agricole complète mais seulement sur une même année civile et la phénologie du blé n'était pas gérée.

Nous avons donc, dans une optique de valorisation de l'avertissement agricole et de l'expérimentation fongicide, décidé de reprendre entièrement la conception et l'organisation du modèle SIMUL.

L'originalité du modèle nouvellement mis en forme, à la différence des systèmes concernant la rouille brune travaillés par ailleurs, est d'être un modèle déterministe, non d'approche statistique, mais basé sur le cycle de l'urédospore de rouille brune. Son ambition est d'être d'utilisation simple, de conception et d'organisation très ouvertes et modulables, ayant une mission d'information et de conseil, permettant de simuler la maladie en temps réel, apportant ainsi une amélioration aux avertissements agricoles.

Ainsi les objectifs de notre travail sont de tenter d'obtenir des simulations satisfaisantes de l'évolution de la rouille brune sur différentes années, sur des variétés de blé dur et de blé tendre et de sensibilités diverses.

\section{MATÉRIEL ET MÉTHODES}

Le modèle EPURE est composé de 2 sous-modèles : un sous-modèle plante et un sous-modèle champignon.

\section{Le sous-modèle plante}

Ce sous-modèle permet d'évaluer, chaque jour, le nombre de sites disponibles pour la rouille brune, c'est-à-dire le nombre de pustules pouvant occuper le feuillage d'un échantillon théorique de 100 tiges de blé (fig 1). Le calcul du nombre s'inspire des travaux de Darles (1980); il est obtenu à partir de l'équation suivante :

$$
N=C \times 96 \times S \times(1-n)
$$

avec $N$ : nombre maximal de sites;

$n$ : proportion de surface foliaire nécrosée;

$C$ : proportion de surface foliaire contaminable;

$S$ : surface de la feuille;

96 : nombre maximal de pustules sur.les 2 faces d'une portion de $1 \mathrm{~cm}^{2}$ de feuille.

Ce sous-modèle effectue premièrement une simulation du stade du blé à partir de la somme de degrés jours en base $0{ }^{\circ} \mathrm{C}$, par cumul depuis le semis, nécessaire à la réalisation des stades clés du développement du blé : levée, épi $1 \mathrm{~cm}$, épiaison, grain laiteux, grain pâteux. II tient compte de la précocité du blé ( 6 groupes de précocité variétale ont été retenus) et de la date de semis (6 périodes ont été envisagées), (d'après Duboin, 1986a).

II évalue ensuite, chaque jour, le nombre de sites potentiels à partir de 2 tables :

- l'une définissant une correspondance entre le stade phénologique et une structure de feuillage : nombre moyen de feuilles présentes et pourcentage moyen de surface contaminable de ces feuilles;

- l'autre définissant le nombre de sites potentiels suivant le stade phénologique.

\section{Le sous-modèle champignon}

Ce sous-modèle ne s'intéresse qu'à la phase épidémique de la maladie en se basant sur le cycle de l'urédospore et comprendra 3 compartiments : contamination, incubation et sporulation (fig 2).

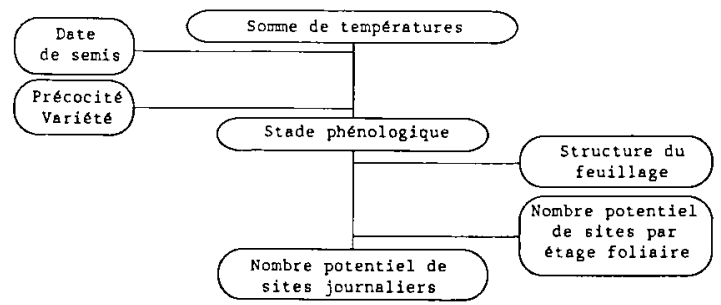

Fig 1. Organigramme du sous-modèle plante. Détermination du stade phénologique à partir de la somme des températures moyennes journalières, en base $0^{\circ} \mathrm{C}$, depuis le semis, en tenant compte de la précocité du blé et de la date de semis. $\grave{A}$ ce stade correspondent une structure foliaire (nombre de feuilles présentes) et un nombre de sites potentiels par étage foliaire, permettant la détermination d'un nombre potentiel de sites journaliers (nombre de pustules pouvant se développer sur le feuillage de 100 tiges de blé). 


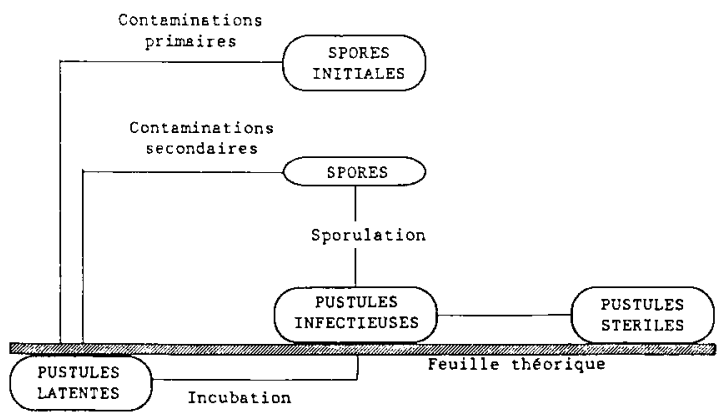

Flg 2. Organigramme du sous-modèle champignon. Ce sousmodèle est basé sur le cycle de l'urédoscope de rouille brune et comprend 3 compartiments :

- un compartiment contamination permettant de calculer, chaque jour, le nombre de pustules latentes représentant le mycélium en incubation dans la feuille de blé, et comptabilisant les spores qui germent et pénètrent à partir des spores provenant soit des pustules infectieuses, soit des spores initiales contaminatrices;

- un compartiment incubation assurant la transformation des pustules latentes en pustules infectieuses;

- un compartiement sporulation assurant la transformation des pustules infectieuses en pustules épuisées.

\section{Le compartiment contamination}

Dans le modèle EPURE, ce compartiment permet de calculer, chaque jour, le nombre de «pustules latentes». Les «pustules latentes» n'ont aucune signification biologique. Nous considérons qu'elles représentent le mycélium en latence de formation de pustules dans la feuille de blé, à partir des spores qui ont germé et pénètrent. Ainsi, le nombre de upustules latentes" correspond au nombre de contaminations réussies.

La température et l'humectation constituent les 2 principaux facteurs limitant la contamination. Pour gérer l'influence de ces 2 paramètres, le modèle utilise la température et l'humectation avec la périodicité trihoraire.

L'effet de la température, par rapport aux températures cardinales définies par Clifford et Harris (1981), est traduit sous la forme d'un facteur température $P C O N T$, variant de 0 à 1 (fig 3).

L'effet de l'humectation est traduit, comme la température, sous la forme d'un facteur humectation $D H U M$, variant de 0 à 1 , suivant le nombre d'heures d'humectation continue dès que le seuil de $4 \mathrm{~h}$ est dépassé (fig 3).

Toutes les $3 \mathrm{~h}$, le nombre d'heures d'humectation est mis en mémoire. Toute période trihoraire dont la durée d'humectation est nulle a pour effet d'annuler la période contaminatrice. Les durées d'humectation entamées la veille sont prises en compte le jour suivant.

Le fait que l'humectation soit un paramètre rarement mesuré dans les stations météorologiques, nous a conduits à utiliser, quand cette variable n'est pas dis-

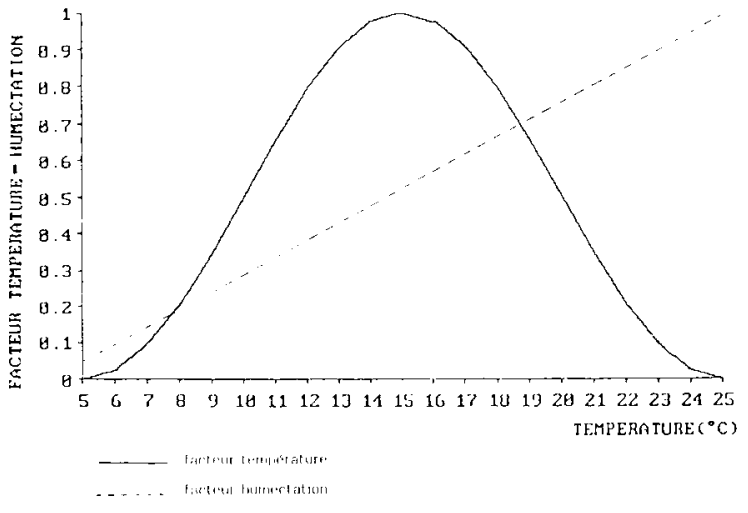

Fig 3. Etfet de la température et de la durée d'humectation sur la contamination. L'effet de la température est traduit sous la forme d'un facteur température, variant de 0 à 1 . C'est une fonction de la température selon la formule : $y=$ $\sin ^{2}(45-9 x)$. L'effet de l'humectation est traduit sous la forme d'un facteur humectation, variant de 0 à 1 dès que le seuil de 4 heures d'humectation continue est dépassé.

ponible, l'hygrométrie, en partant de l'hypothèse que toute valeur d'hygrométrie $\geq$ le seuil de $84 \%$ correspond à une durée d'humectation de $3 \mathrm{~h}$.

La comparaison d'un indice contamination (I) déterminé à partir de l'humectation et de la température $(I=P C O N T \times D H U M)$ et à partir de l'hygrométrie et de la température ( $I=P C O N T \times H Y G R O)$, nous a permis de montrer que les cumuls des indices donnent des valeurs pratiquement identiques, qu'on utilise l'humectation ou l'hygrométrie.

Ainsi, le modèle EPURE permet de déterminer, chaque jour, un nombre RLAT de nouvelles pustules latentes:

$R L A T=(S T A+S P O R E) \times[(A L L-L A T-I N F-S T E R)$ I ALL × PCONT × DHUM]

et d'en déduire le stock au jour $\mathrm{j}$ :

$$
L A T_{(j)}=L A T_{(j-1)}+R L A T_{(j)}-R I N F_{(j)}
$$

avec :

$S T A=$ nombre théorique de spores initiales contaminatrices nécessaires à l'initialisation du système;

$S P O R E=$ nombre de spores contaminatrices issues des pustules infectieuses et arrivant sur la feuille de blé;

$A L L=$ nombre de sites disponibles sur le feuillage de l'échantillon de 100 tiges de blé, variable suivant le stade phénologique;

$L A T, I N F, S T E R=$ stock de "pustules latentes", infectieuses (sporulantes) et épuisées au jour j;

$R I N F=$ «nouvelles pustules infectieuses" au jour $j$;

$P C O N T=$ facteur température;

$D H U M=$ facteur humectation. 


\section{Le compartiment incubation}

Le compartiment incubation assure la transformation des "pustules latentes" en pustules infectieuses (sporulantes). Le passage d'un état à l'autre dépend de la température moyenne journalière; il est géré par la fonction Boxcare-train. Celle-ci a pour rôle d'assurer le transfert des pustules, qui se situent dans un état donné, d'un compartiment à l'autre par trains successifs (De Wit et al, 1974; Rijsdijk, 1975; Teng et al, 1980). La transformation des pustules latentes en pustules infectieuses ne se fait donc pas de manière brusque mais par paliers, de façon progressive. Le nombre de paliers est fixé à 5 .

Suivant la température moyenne journalière, la période d'incubation correspondante et le nombre de paliers, nous pouvons définir une vitesse journalière de passage d'un état à l'autre et la quantité de pustules latentes transformées en pustules infectieuses lorsque $20 \%$ de la phase d'incubation sont accomplis ( $1 / 5$ de la phase d'incubation).

Ainsi, le modèle permet de déterminer un nombre de pustules infectieuses :

$$
I N F_{(j)}=I N F_{(j-1)} \times(\mathfrak{t}-M O D I F)+R I N F_{(j)}-R S T E R_{(j)}
$$

avec :

MODIF = pourcentage de réduction du nombre de sites suivant le stade phénologique pour tenir compte du dessèchement par sénescence des étages foliaires inférieurs;

RSTER $=$ nouvelles pustules épuisées au jour $j$ dont le stock journalier correspond à STER.

La période d'incubation suivant la température, introduite dans le modèle, est rapportée dans la figure 4 (d'après Gassner et Apple, in Richard, 1984). Cette période commence à la pénétration et se termine à l'apparition des premiers symptômes.

\section{Le compartiment sporulation}

Ce compartiment rend compte du rythme et du flux de libération des urédospores à partir des pustules infectieuses et assure donc la transformation des pustules

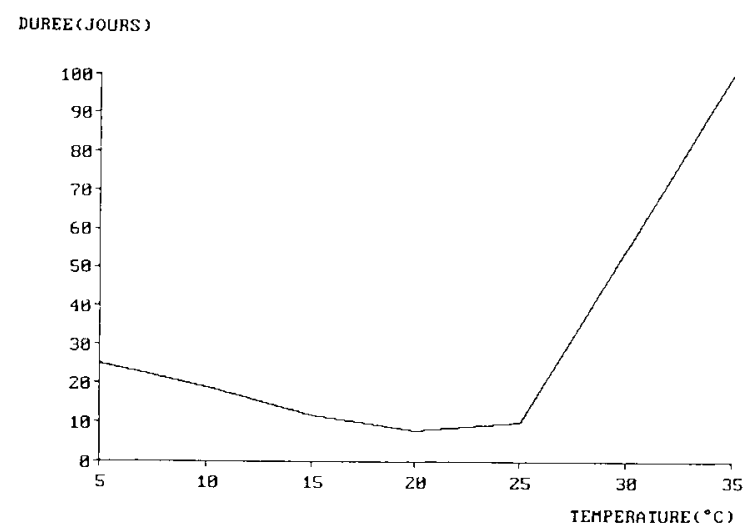

Fig 4. Effet de la température sur la durée d'incubation. infectieuses en pustules épuisées (vidées); passage également géré par la fonction Boxcar-train, qui intègre la durée de sporulation suivant la température (fig 5) (d'après BOVA, 1983) :

$$
S T E R_{(j)}=S T E R_{(j-1)} \times(1-M O D I F)+R S T E R_{(j)}
$$

avec $: M O D I F=$ pourcentage de réduction du nombre de sites suivant le stade phénologique pour tenir compte du dessèchement par sénescence des étages foliaires inférieurs;

$R S T E R$ = «nouvelles pustules épuisées» au jour j.

II se produit une certaine compensation entre la durée de sporulation et le nombre total de spores produites au cours d'un cycle de sporulation. Zadoks et Schein (1979) parlent de strategy of life : des conditions de températures favorables permettent une brève durée de sporulation avec production importante d'urédospores par jour, alors que de mauvaises conditions impliquent une période de sporulation allongée avec une faible quantité de spores produites; la durée de libération est allongée, le nombre de spores reste inchangé (Mehta et Zadoks, 1970).

Ceci nous a conduit à introduire la notion de spores dans le modèle, en partant de l'hypothèse qu'à la température optimale de sporulation $\left(20^{\circ} \mathrm{C}\right)$, le facteur rendant compte de la quantité de spores libérées (COEFF.SPO. variant de 0 à 1) est également optimal.

Ainsi, le nombre de spores issues des pustules infectieuses est obtenu à partir de l'équation :

$$
\operatorname{SPORE}_{(j)}=I N F_{(j)} \times \text { COEFF.SPO } \times \text { DMFR }
$$

avec : INF = stock de pustules infectieuses au jour j; COEFF.SPO = coefficient rendant compte de la quantité de spores libérées journellement qui varie de 0 à 1 et est obtenu par la formule : COEFF.SPO = MIN.SPODU/PINF où MIN.SPODU est la durée de

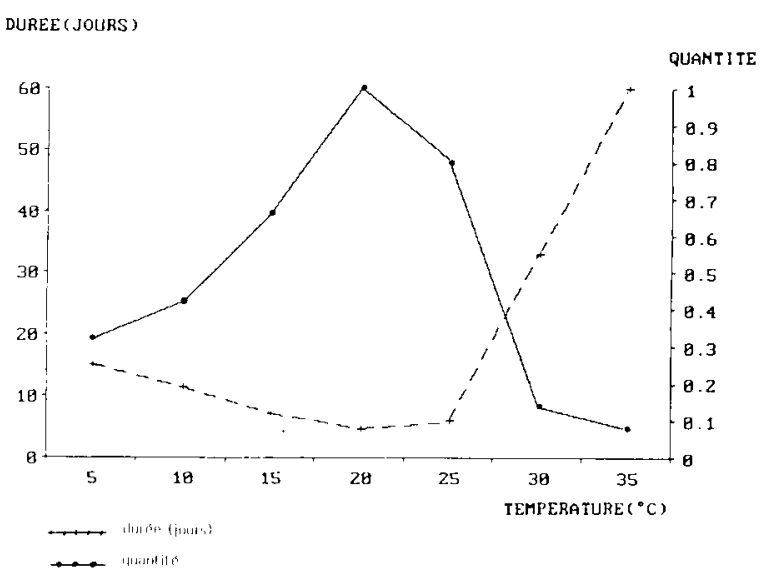

Fig 5. Effet de la température sur la durée de sporulation et la quantité de spores libérées. L'effet de la température sur la quantité de spores libérées journellement est traduit sous la forme d'un facteur variant de 0 à 1 . À la température optimale de sporulation, la quantité de spores libérées est également optimale. 
sporulation la plus courte $(4,8$ j) et PINF la durée de sporulation suivant la température;

$D M F R=$ taux de multiplication journalier permettant de calculer le nombre de spores issues d'une pustule sporulante. Ce taux tente de rendre compte du nombre de spores efficaces par rapport au nombre de spores produites.

Ce taux de multiplication est maintenu constant durant toute la campagne.

\section{RÉSULTATS : VALIDATION DU MODĖLE}

Afin de réaliser la validation du modèle EPURE, nous avons utilisé certaines années historiques d'évolution de la rouille brune tirées, d'une part, des essais d'homologation des produits phytosanitaires (parcelles témoins dont les données nous ont été transmises par le SPV MidiPyrénées) et d'autre part, d'observations que nous avons réalisées.

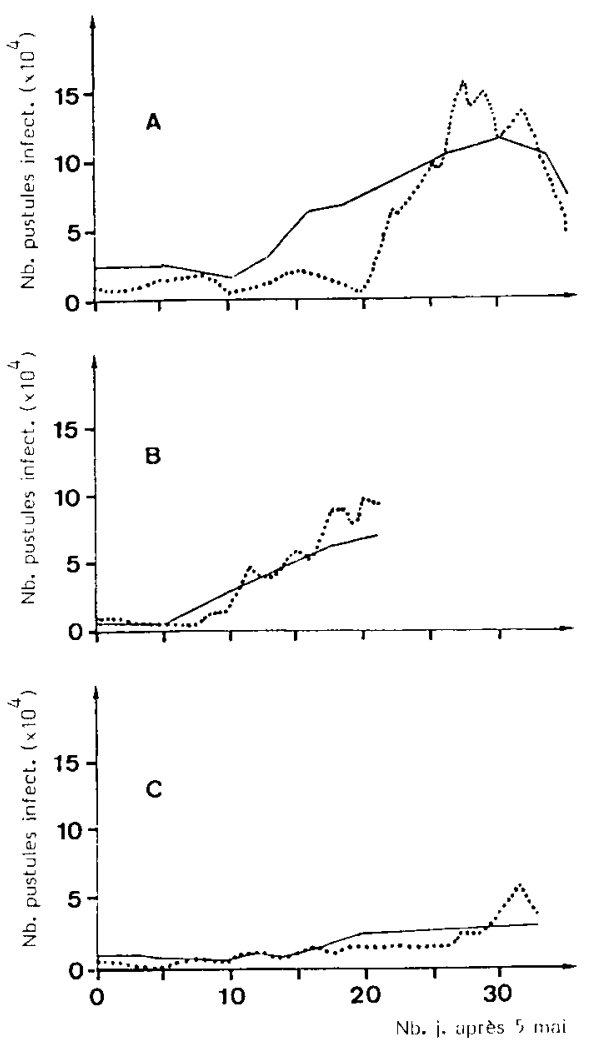

pustules infectieuses réclies

A - Rerneville (31), var, Talenh, $5 \mathrm{emis}$ 5.12.1941

B - Auzeville (31), var. Caston, senis 9.11.1982

C - Muntrabe (31), var. Talent, semis 9.11 .1982

D - Balma (31), var. Calpdur, scmis 12.11.198'

[ - Avezan (32), var. Castan, scmis 28.11.1987

F - Dremil Lalane (31), var. Primadur, semis 5.11.1487
Pour chaque cas étudié, nous avons dressé des graphiques (fig 6) où figurent le nombre de pustules infectieuses issu d'un comptage au champ (comptage du nombre de pustules infectieuses sur les faces supérieures et inférieures des feuilles de 100 tiges de blé) et le nombre de pustules en sortie du modèle. Cette validation du modèle a été réalisée sur 4 campagnes agricoles (entre 1981-1982 et 1987-1988) et a concerné 6 parcelles aussi bien de blé dur que de blé tendre.

\section{DISCUSSION}

Présentation de l'évolution de la rouille brune

Certaines années, comme en particulier les campagnes 1981-1982 (fig 6A), 1982-1983
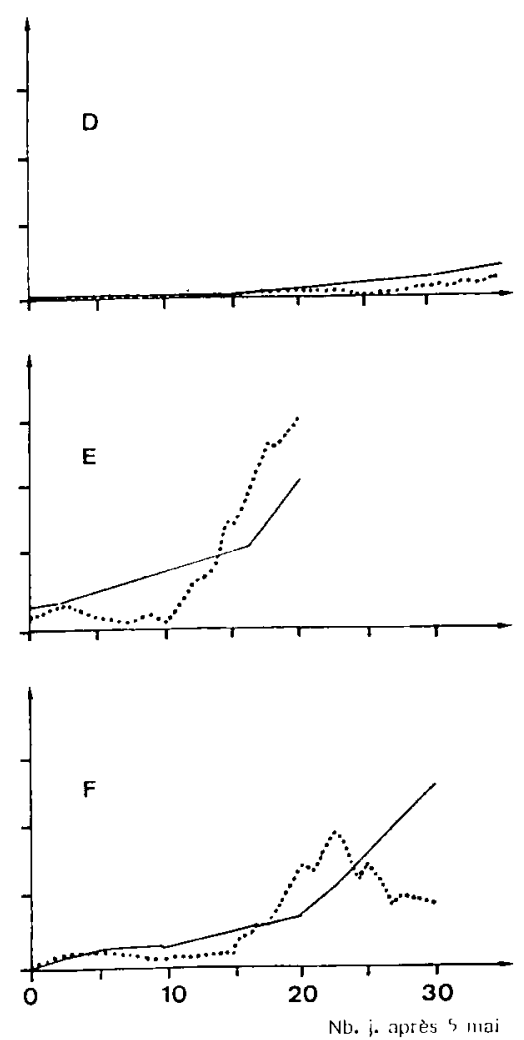

Fig 6. Comparaison du nombre de pustules infectieuses réelles et simulées. 
(fig 6B) et 1987-1988 (fig 6E, F) se caractérisent par une explosion précoce de la maladie, mais avec des intensités d'attaque variables.

La douceur de l'hiver conditionne de façon notable la précocité de l'épidémie, mais si le printemps est marqué par des périodes de froid, ce démarrage rapide est pondéré. Cela conduit à des situations où la rouille brune est présente tôt en saison mais en faible quantité (palier des courbes $C$ et $F$, fig 6 ).

Par contre, d'autres campagnes, comme en particulier l'année 1985-1986 (fig 6D), se caractérisent par une manifestation tardive de la rouille brune avec de faibles intensités d'attaque.

\section{Analyse et interprétation des données proposées par le modèle}

D'une manière générale, les simulations indiquent correctement le début des explosions de la maladie, quelles que soient les années considérées.

Dans le cas où le dessèchement provoqué par la sécheresse a stoppé le développement de la rouille brune (d'où une diminution du nombre de pustules infectieuses), le modèle réagit correctement aux températures élevées en combinaison avec une absence d'eau (fig 6A).

Le modèle traduit également correctement, pour une même année, des conditions météorologiques différentes suivant la localisation des parcelles. C'est le cas pour l'année 1982-1983, où le nombre de pustules simulées est moins important dans la parcelle située à Montrabe (fig $6 \mathrm{C})$ que dans celle située à Auzeville (fig 6B). De la même façon, le modèle simule correctement, pour l'année 1987-1988, le démarrage plus précoce de l'épidémie dans la parcelle située dans le Gers (fig $6 \mathrm{E}$ ) que dans celle en Haute-Garonne (fig 6F).

À partir de fin mai-début juin, ce nombre de pustules est généralement quelque peu surestimé par rapport à l'observation de terrain du fait d'un taux de multiplication DMFR maintenu constant durant toute la campagne.

Malgré tout, il n'y a pas d'années véritablement aberrantes; certaines simulations sont un peu moins fiables que d'autres, comme en particulier celles concernant la campagne 1981. 1982.

II aurait été souhaitable de valider le modèle sur un nombre plus important d'années, mais cela n'a pas été possible car nous nous sommes heurtés dès le début de ce travail à plusieurs problèmes concernant le choix des parcelles et les données récoltées.

Concernant le choix des parcelles, les essais d'homologation, qui nous servent de référence, n'ont pas été réalisés, pour les années étudiées, sur la même exploitation mais sur des parcelles différentes et éloignées. Ceci implique des conditions agronomiques diverses et ne facilite pas les comparaisons d'évolution de la rouille brune pour les années successives et la validation du modèle. D'autant que l'influence des conditions agronomiques n'est prise en compte que partiellement dans les calculs d'EPURE. D'autre part, les parcelles ne sont pas toutes proches d'un même poste météorologique complet (données d'hygrométrie et/ou d'humectation absentes).

Concernant les données récoltées, on observe que :

- pour certains essais, les renseignements sur la date de semis et/ou la variété de blé semée ne sont pas toujours mentionnés, d'où l'impossibilité d'exploiter ces essais;

- les notations des essais, basées sur un comptage de pustules suffisamment précis sont rares, compte tenu des protocoles d'observation non adaptés (estimation le plus souvent de surfaces atteintes et non du nombre de pustules);

- ces notations s'effectuent à l'œil nu, d'où une nette sous-estimation du nombre de pustules. De plus, elles ne prennent en compte que la face supérieure des feuilles, alors que le modèle détermine un nombre de pustules présentes sur les 2 faces;

- le modèle donne un nombre global de pustules infectieuses pour tous les étages foliaires simultanément, alors que les notations s'effectuent feuille par feuille et ne concernent, la plupart du temps, que les étages supérieurs.

Ainsi, bien que le modèle EPURE donne aujourd'hui des résultats plutôt satisfaisants, un certain nombre d'améliorations pourraient lui être apportées.

Concernant le sous-modèle plante :

- l'estimation des stades par le modèle semble suffisante pour ne pas impliquer d'erreur notable dans la suite des calculs. Cependant, il conviendrait d'introduire un facteur "pluie" pour mieux évaluer la date de levée. En effet, la date de levée n'est actuellement estimée qu'en tenant compte de la température moyenne journalière. 
Or une absence de précipitation pendant la période de semis peut retarder de façon notable la levée. Pour germer, une semence de céréale a besoin d'eau; un grain en absorbe $25 \%$ de son poids (Duboin, 1986);

- une autre amélioration viserait à mettre en place un sous-modèle plante qui gèrerait la croissance de chaque étage foliaire et non pas une évolution globale de la phénologie du blé.

Concernant le sous-modèle champignon :

- il serait souhaitable de pouvoir utiliser l'humectation dans toutes les situations. II conviendrait, pour cela, d'affiner les équations de transfert pour simuler l'humectation soit à partir de l'hygrométrie trihoraire ou des données journalières;

- le nombre de spores initiales contaminatrices, nécessaires à l'initialisation du système (STA), est fixé à une valeur constante. II conviendrait d'étudier l'intérêt d'une pondération de ce paramètre, suivant la prévalence de la rouille brune, l'année précédente, dans la région considérée;

- le taux de multiplication DMFR est également maintenu, dans notre modèle, à une valeur constante durant toute la campagne. II serait nécessaire d'étudier l'intérêt d'une variation de ce paramètre en cours de saison;

- le nombre de sites disponibles pour la rouille brune varie suivant le stade phénologique. Mais une gestion de ce nombre de sites, par étage foliaire et non globalement par tige, permettrait une meilleure appréhension des phénomènes de dessèchement, et donc de réduction du nombre de pustules en cours de campagne. Cette amélioration serait d'autant plus intéressante que les seuils de nuisibilité sont basés à ce jour sur l'importance de la maladie au niveau des étages foliaires.

Enfin, EPURE est également dimensionné pour être utilisé en tant que modèle d'aide à la décision à la parcelle, de par sa conception et son organisation très ouvertes et modulables, dans le cadre, notamment, de l'interprétation des résultats d'expérimentations de produits phytosanitaires et du raisonnement des interventions fongicides.

II conviendrait, pour améliorer cette utilisation, de prendre en compte quelques autres paramètres agronomiques et culturaux, tels la fertilisation azotée et l'effet des fongicides.

En effet, actuellement, le modèle EPURE ne peut simuler l'évolution du pathogène que dans des parcelles témoins, caractérisées par l'absence de traitement. La prise en compte d'applications fongicides impliquerait de faire tendre vers 0 le taux de multiplication DMFR tant que le produit appliqué est actif, donc de réduire le nombre de spores infectieuses (SPORE). Ceci nécessiterait, en outre, de tenir compte de l'efficacité des différents fongicides et de leur période de rémanence en considérant que l'épidémie peut redémarrer une fois que l'activité toxique du produit est terminée. C'est l'ambition que nous avons et qu'il conviendrait de poursuivre afin de permettre d'assurer une meilleure conduite de la lutte contre la rouille brune du blé.

\section{RÉFÉRENCES}

Benizri E (1989) Mise au point d'un modèle de simulation et de prévision de la rouille brune du blé. Thèse Doct Sci Agron INPT, $199 \mathrm{p}$

Bova F (1983) Propositions pour l'amélioration du système d'avertissements agricoles "Posypre» sur les septorioses et la rouille brune du blé d'hiver. Mémoire ENSSAA

Burleigh JR, Eversmeyer MG, Roelfs AP (1972) Development of linear equations for predicting wheat leaf rust. Phytopathology 62, 944-946

Caron D (1988) Évolution des épidémies de rouille brune (Puccinia recondita Rob et Desm) et prévision des traitements dans le sud de la France. ANPP $2^{\ominus}$ Conf Int maladies Plant Bordeaux 8-9-10 nov 1988

Chester KS (1946) The nature and prevention of cereal rusts as exemplified in the leaf rust of wheat. Chron Bot Co, Waltham, Mass

Clifford BC, Harris RG (1981) Controlled environment studies of the epidemic potential of Puccinia recondita $\mathrm{fsp}$ tritici of wheat in Britain. Trans $\mathrm{Br} \mathrm{Mycol}$ Soc 77, 351-358

Darles M (1980) Modélisation des épidémies de rouille brune sur blé tendre. Mém ENSAT

De Wit CT, Goudriaan J (1974) Simulation of ecological processes. Pudoc, Wageningen

Duboin G (1986a) Adaptation des variétés aux petites régions et à la date de semis. Méthode de choix d'un type de variété. Perspect Agric, HS, 38-44

Duboin $\mathrm{G}$ (1986b) Relations entre stades de développement et techniques culturales. Perspect Agric HS, 28-37

Knudsen GR, Spurr HW, Johnson CS (1987) A computer simulation model of Cercospora leaf spot of peanut. Phytopathology 77, 1118-1121

Mehta YR, Zadoks JC (1970) Uredospore production and sporulation period of Puccinia recondita $\mathrm{fsp}$ triticina on primary leaves of wheat. Neth J Plant Pathol 76, 267-276

Poilane PH (1982) Prévision de la rouille brune dans le sud de la France. Mém ENSAT

Richard C (1984) Prévision des épidémies de rouille brune dans le Lauragais et méthode de lutte. Mém ENSAT 
Rijsdijk FH (1975) A simulator of yellow rust of wheat. Bull Rech Tech Agron Gembloux, 411-418

Teng PS, Blackie MJ, Close RC (1980) Simulation of the barley leaf rust epidemic: structure and validation of Barsim. I. Agric Syst 5, 85-103
Waggoner PE, Norwell WA, Royle DJ (1980) The low and the minimum and the relation between pathogen, weather, and disease. Phytopathology 70, 85-103

Zadoks JC, Schein RD (1979) Epidemiology and plant disease management. Oxford Univ Press, New York 